FERMILAB-TM-2637-DI

October 2016

\title{
Accelerator/Experiment Operations - FY 2016
}

\begin{abstract}
A. Blake, M. Convery, S. Geer ${ }^{1}$, D. Geesaman, D. Harris, D. Johnson, K. Lang, K. McFarland, M. Messier, C. D. Moore, D. Newhart, P. E. Reimer, R. Plunkett, M. Rominsky, M. Sanchez, J.J. Schmidt, P. Shanahan, C. Tate ${ }^{2}$, J. Thomas, D. Torretta, M. Wetstein
\end{abstract}

\section{Introduction}

This Technical Memorandum summarizes the Fermilab accelerator and experiment operations for FY 2016. It is one of a series of annual publications intended to gather information in one place. In this case, the information concerns the FY 2016 NOvA, MINOS+ and MINERvA experiments using the Main Injector Neutrino Beam (NuMI), the MicroBooNE experiment and the activities in the SciBooNE Hall using the Booster Neutrino Beam (BNB), and the SeaQuest experiment, LArIAT experiment and Meson Test Beam activities in the $120 \mathrm{GeV}$ external switchyard beam (SY120).

Each section was prepared by the relevant authors, and was then edited for inclusion in this summary.

Accelerator Operations (M. Convery, D. Johnson, D. Newhart)

The low-energy and high-energy neutrino beams, the SeaQuest beam, and the LArIAT and Test Beams in the Meson area were brought into operation after the summer 2015 accelerator shutdown. The startup occurred on October $15^{\text {th }}$, determined by the schedule for the new Booster anode supplies, and ran until the present shutdown which began July $29^{\text {th }}$. The main summer 2016 shutdown drivers are Recycler collimator installation and the second of three phases of vacuum TSP to ion pump upgrades. Other jobs include the installation of $2.5 \mathrm{MHz} \mathrm{RF}$ in the Recycler for the Muon Campus, preparation for adding two more Booster RF cavities under the Proton Improvement Plan (PIP), and enclosure modifications to allow beam to BNB while accessing roughly two-thirds of the Main-Injector tunnel.

The high-energy neutrino beam was scheduled for beam delivery for 39 weeks. NuMI beam power was ramped up over the year. The number of batches slip-stacked in the Recycler was increased in steps. At each step, intensity was increased while tuning for efficiency and losses. $700 \mathrm{~kW}$ was successfully demonstrated for one cycle with consistent running around $550 \mathrm{~kW}$ prior to the shutdown. Regular $700 \mathrm{~kW}$ operation is expected after the summer 2016 shutdown when Recycler collimators are in place. There were 5983 hours of high-energy operation in FY 2016.

${ }^{1}$ Editor

${ }^{2}$ Administrative support

Operated by Fermi Research Alliance, LLC under Contract No. DE-AC02-07CH11359 with the United States Department of Energy. 
The Booster Neutrino Beam exceeded expectations due to the quick ramp up in flux facilitated by the ability to run at $15 \mathrm{~Hz}$, thanks to PIP, as well as increased intensity. About 5891 hours of beam was delivered to the MicroBooNE experiment. The approval of a new Booster shielding assessment is needed in order to run at similar flux levels in FY 2017 while also running NuMI at $700 \mathrm{~kW}$.

Beam delivery to SeaQuest, including commissioning time, was 4327 hours with $1.29 \times 10^{18}$ protons delivered. The slow spill beam had the desired intensity and good extraction efficiency.

The Fermilab Test Beam Facility was scheduled for beam for $\sim 39$ weeks of the 52-week period. Beam delivery started on October $24^{\text {th }}$ and continued until July $29^{\text {th }}$. A total of $5.82 \times 10^{15}$ protons were delivered. The facility operated for 1917 hours driven by user requests.

The LArIAT experiment located in the MCenter beam restarted operation in February. Beam was delivered to the experiment for 2748 hours as requested, with $4.6 \times 10^{13}$ protons delivered.

The following plots summarize the beam delivery in FY 2016.

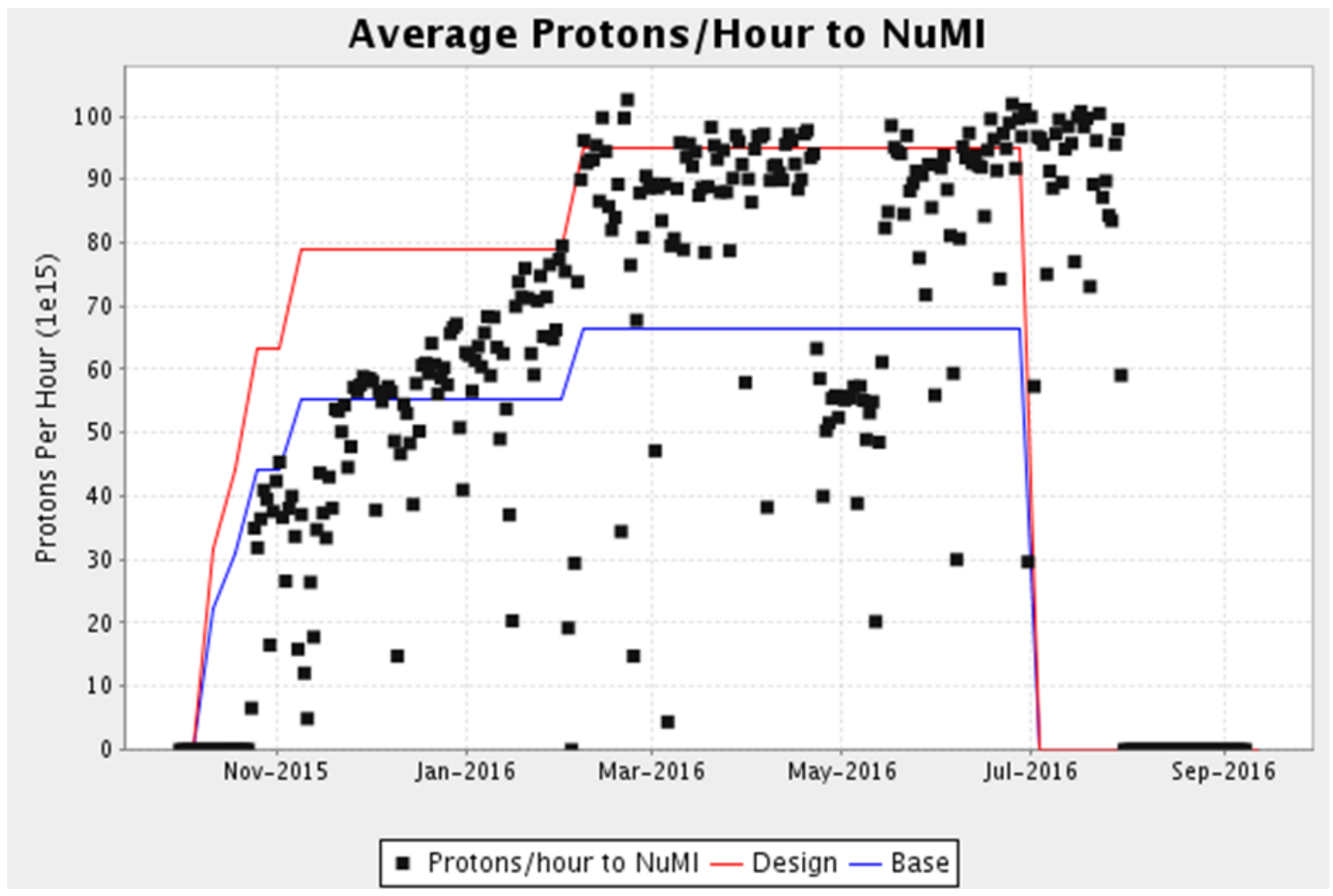


FY16 Integrated Beam to NuMI
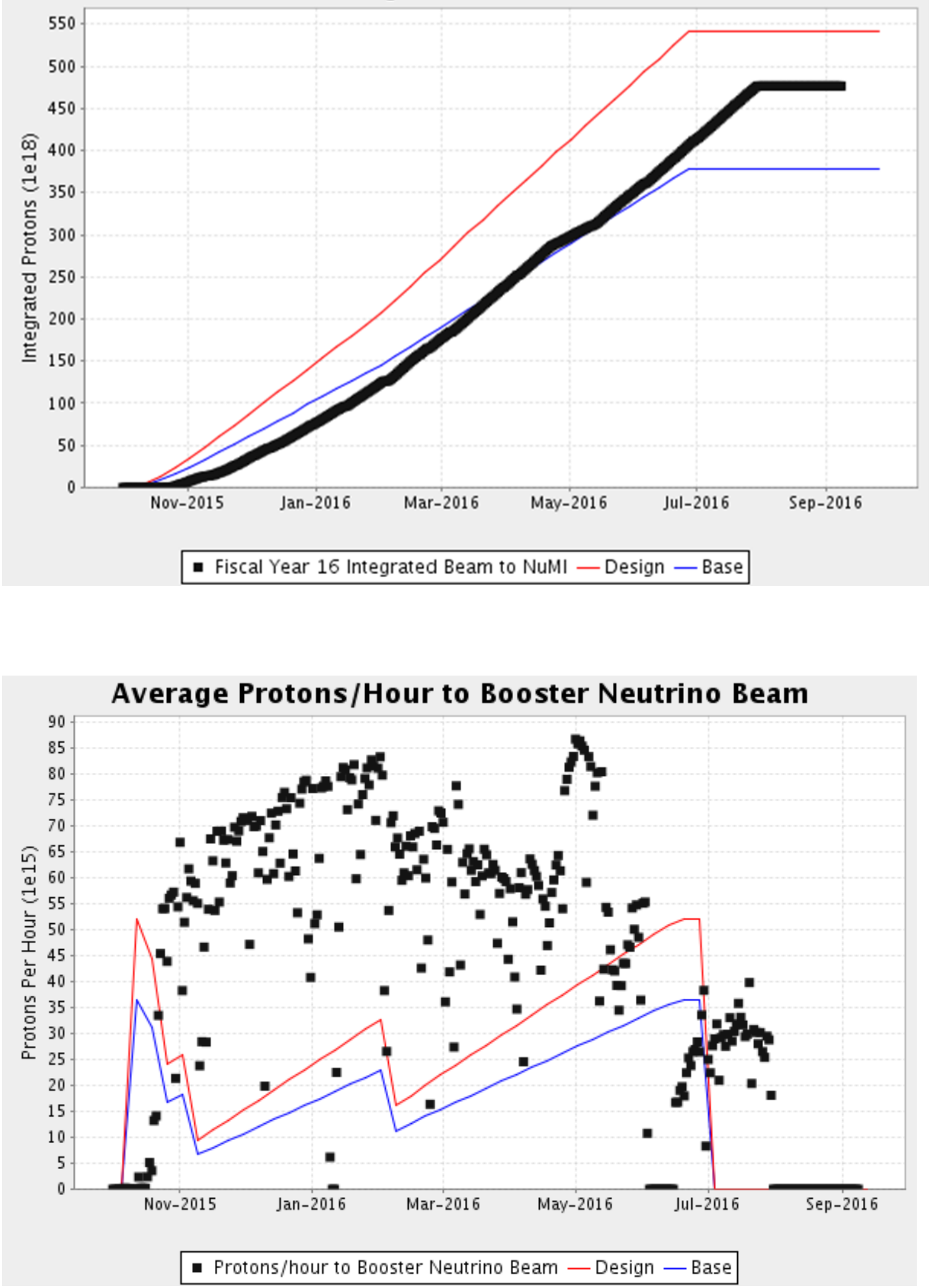


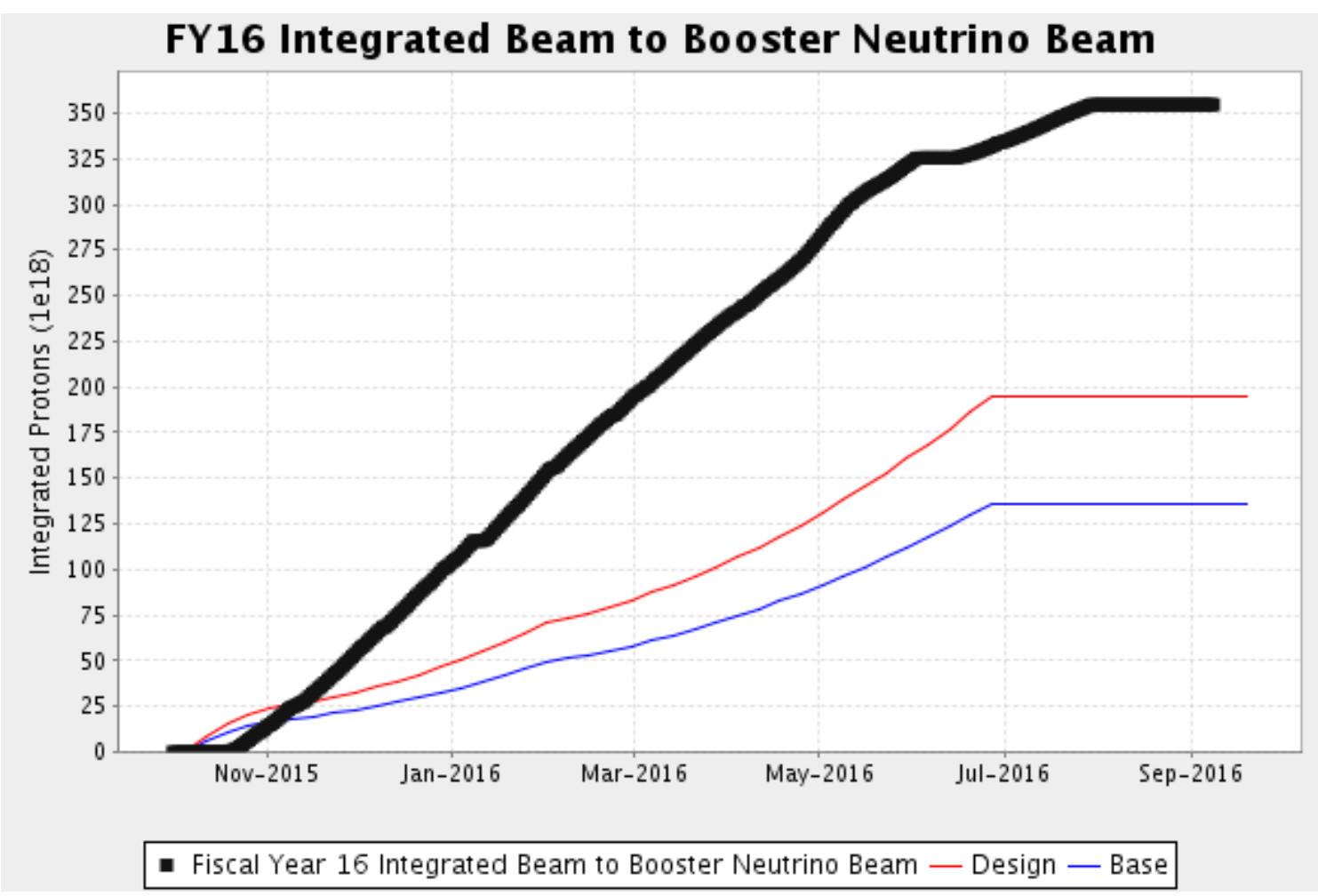

MINOS+ Report (A. Blake, K. Lang, R. Plunkett, J. Thomas, and D. Torretta)

MINOS+ concluded its approved 3 years of data taking at the end of June 2016. A total of $4.1 \times 10^{20}$ POTs for the FY 2016 running period increased the MINOS+ 3-year total to $9.37 \times 10^{20}$. This corresponds to $\sim 50 \%$ of the original request (based on an assumed $700 \mathrm{~kW}$ NuMI beam) and will enable the MINOS+ Collaboration to accomplish their scientific goals. The collaboration is not requesting continued running.

After June $29^{\text {th }}$, the machine switched to reverse horn current mode. MINOS+ personnel maintained the production processes through the end of antineutrino running in summer 2016. These processes have now been handed to MINERvA for the MINOS near detector, which serves as a muon spectrometer for that experiment.

The MINOS detectors performed well in FY 2016, with only minor problems. Figures M-1 and M-2 show the uptime performance of the two detectors.

The physics analysis is ongoing with 7 papers so far this year submitted to journals. Most recently, the MINOS sterile analysis has been accepted for publication together with one from Daya Bay and a joint paper which has been awarded the PRL "editors pick" and will be published in the October $6^{\text {th }}$ edition. The analysis rules out a large region of parameter space where a sterile neutrino might be lurking. Figure M-3 shows the bottom line. 


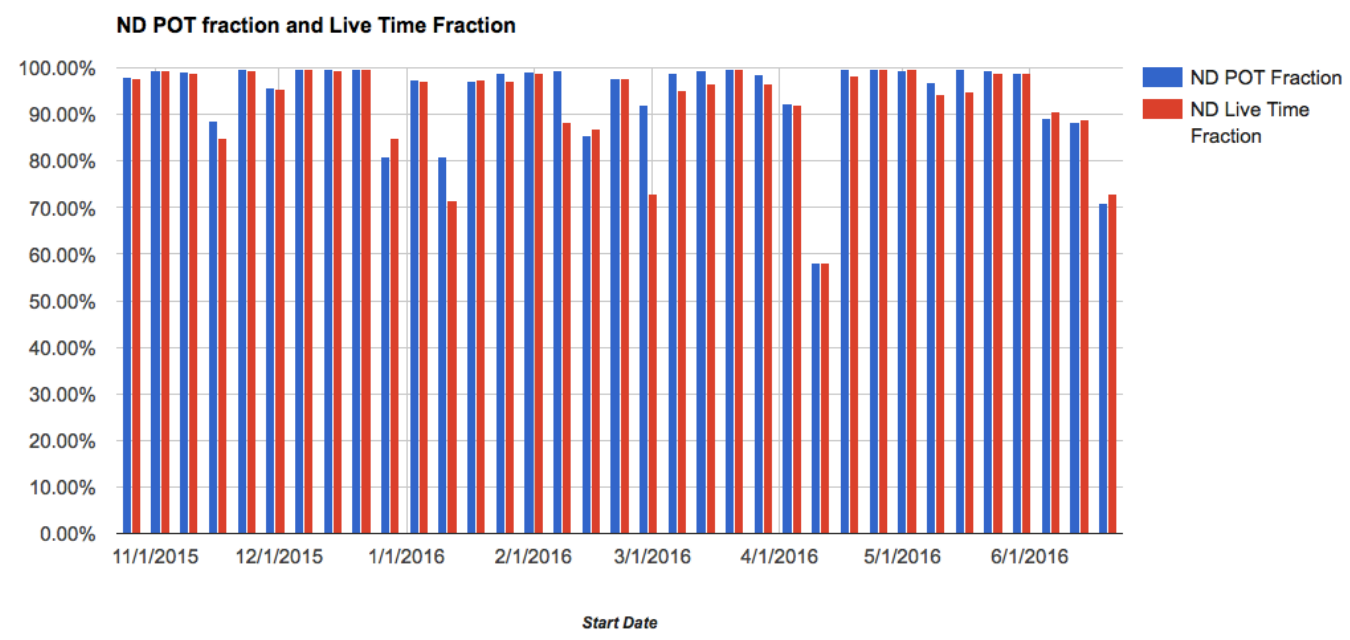

Figure M-1: Live time performance of the MINOS near detector in FY2016. Overall $94.2 \%$ of delivered POT were collected by the detector.

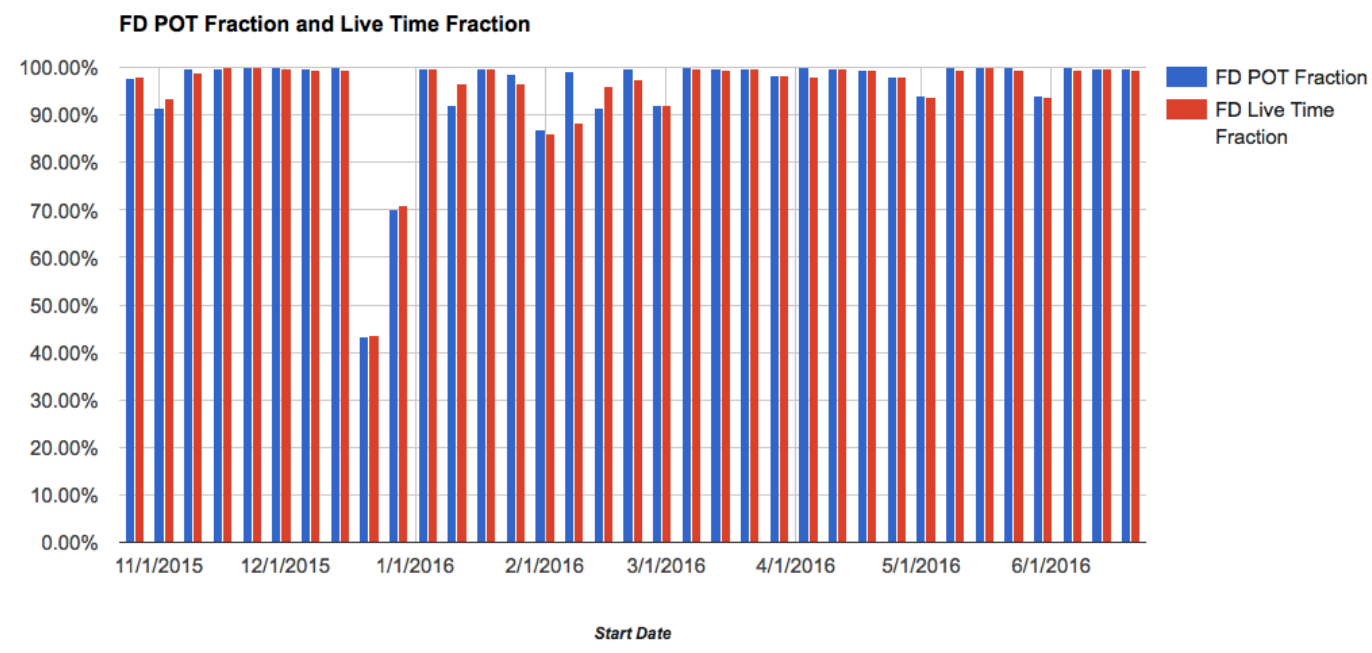

Figure M- 2: Live time performance of the MINOS far detector in FY2016. Overall 95.5\% of the delivered POT were collected by the detector. The lower performance in January 2016 was due to a problem with the GPS system used for detector timing.

The MINOS/MINOS+ collaboration would like to thank the accelerator division at FNAL for their great work over the years.

At the Soudan Underground Laboratory, the MINOS far detector is now being decommissioned. The cosmic ray veto shield will be sent to FNAL to be repurposed as a cosmic ray tagger for ICARUS. Other equipment will be returned to FNAL for repurpose or appropriate disposal. A formal decommissioning process for the MINOS detector steel and scintillator has been initiated. 


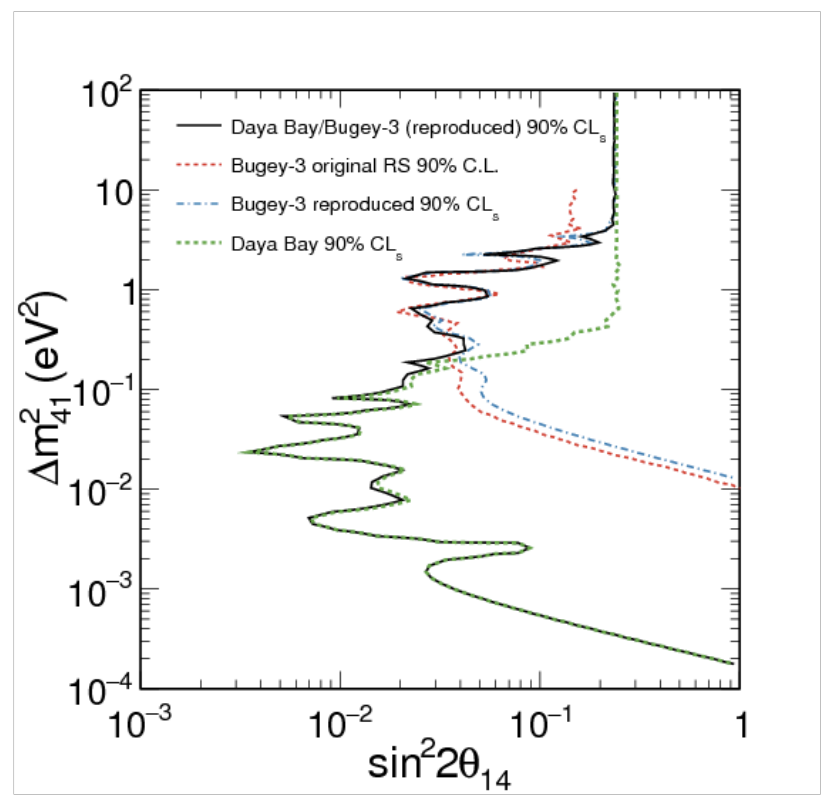

Figure M- 3: Excluded region of sterile-neutrino parameter-space.

NOvA Report (M.D. Messier and P. Shanahan)

NOvA completed its second year of operations in FY 2016, published papers on its first oscillation results, updated both its muon neutrino disappearance and electron neutrino appearance results, and presented its first sterile neutrino search result. Three Ph.D.'s were awarded based on NOvA research in FY 2016, bringing the total to-date to 12.

The experiment continued to staff data-taking shifts $24 \times 7$ regardless of beam status, with experts in data acquisition, detector controls, power supplies, and avalanche photo diode (APD) operations on-call $24 \times 7$. Shifts took place at one of 12 Remote Operations Centers (ROC) including the ROC-West at Fermilab, with ROCs at William \& Mary, Sussex (UK), Goais (Brazil), South Carolina, and Colorado State having come online in FY 2016. A team of 3 Run Coordinators serving in a rotation oversaw daily operations and coordinated activities of the shifters, experts, and support staff. The maintenance and operation of the far detector, and of the Ash River Laboratory, were performed by a crew employed by the University of Minnesota under budget and safety oversight from Fermilab, and in coordination with the collaboration operations team.

The far detector operating efficiency was generally compared with the efficiency in FY 2015. Improvements to data acquisition software in January and March increased the uptime of the detector, and permitted the use of the full buffer/trigger farm capacity for the first time. Overall beam-weighted uptime (the fraction of protons delivered to the NuMI target for which the detector was taking data) was $96.4 \%$ for the fiscal year. Approximately 200 Front End Board/Thermal Electric Cooler Controller (FEB/TECC) units, out of 10752, were swapped due to various levels of performance issues during FY 2016. Thirty-six APDs were swapped. All but about $0.06 \%$ of the FEB/TECCs are typically active during data-taking. 


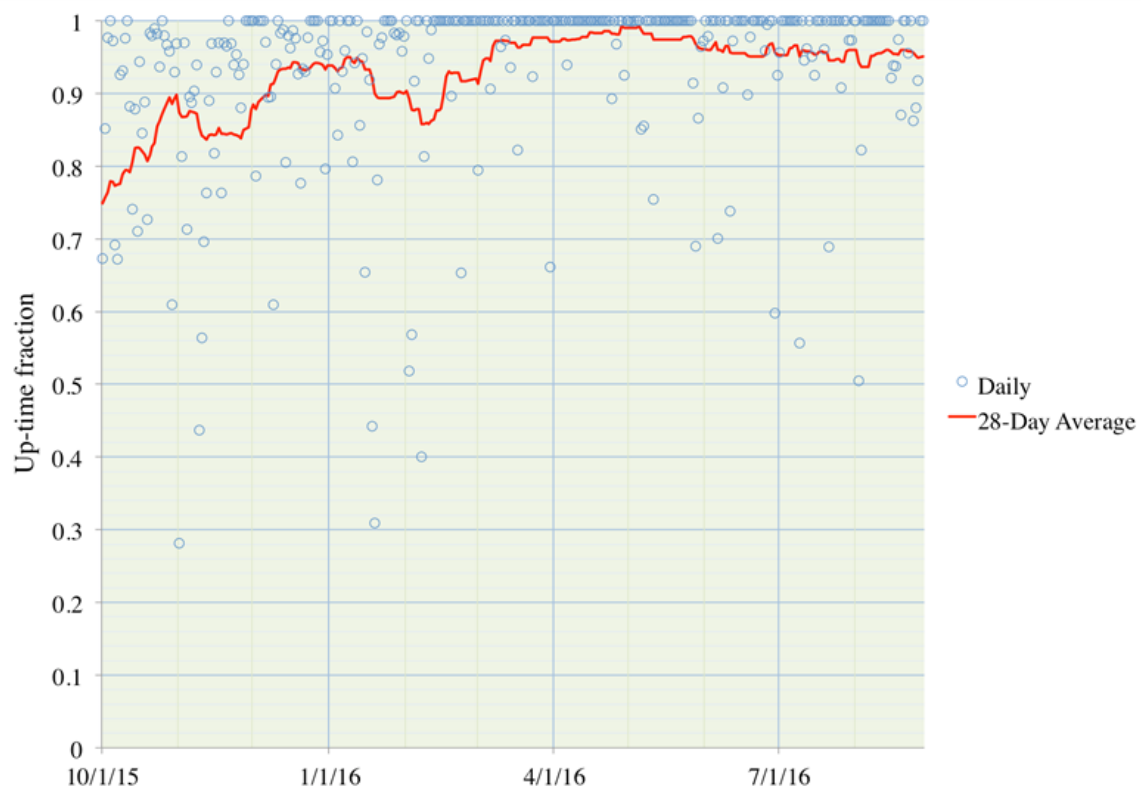

Figure N-1: The NOvA far detector uptime in FY16. This shows the daily (points) and 28-day (red line) average uptime of the NOvA detector systems including planned downtimes for maintenance during beam-off times. Overall the detector operated $>93 \%$ of the time during FY16, with beam-weighted uptime of $96.4 \%$.

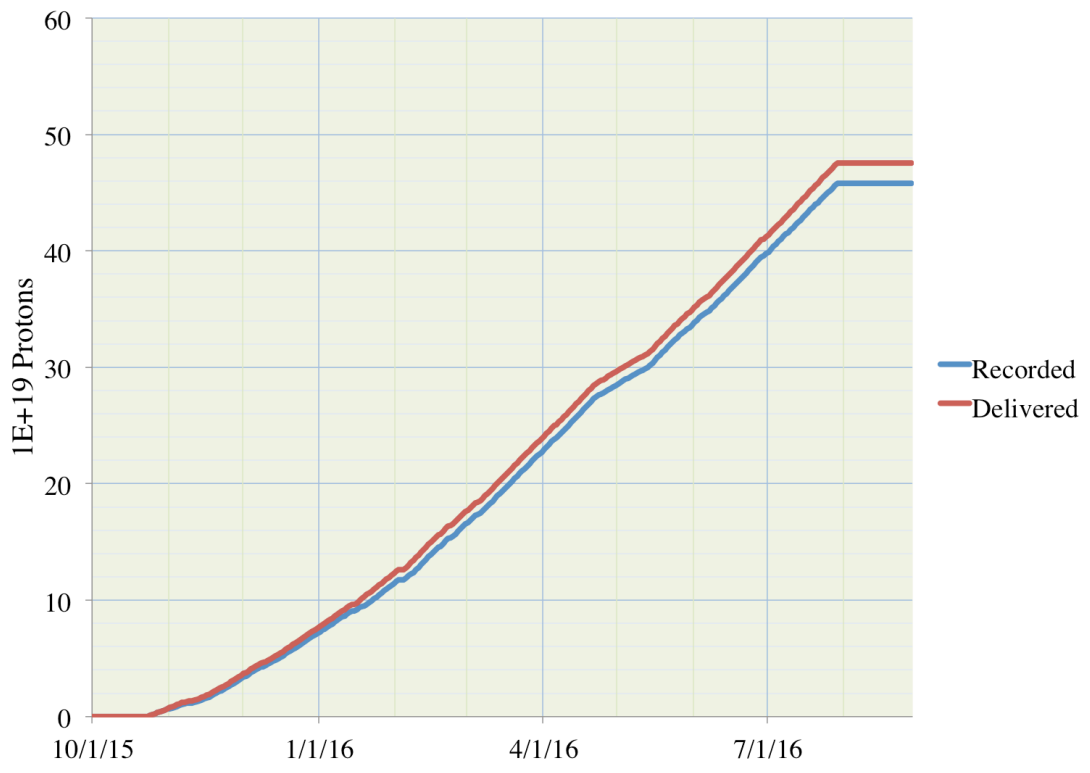

Figure N-2: The total protons on target delivered (red) and recorded (blue) by the NOvA far detector in FY16. The far detector recorded data for 4.58E20 of the 4.75E20 protons delivered to the NuMI target (96.4\%). The NuMI beam was operated in anti-neutrino mode for the last 0.6E20 protons delivered in FY16, starting June 29, 2016. 


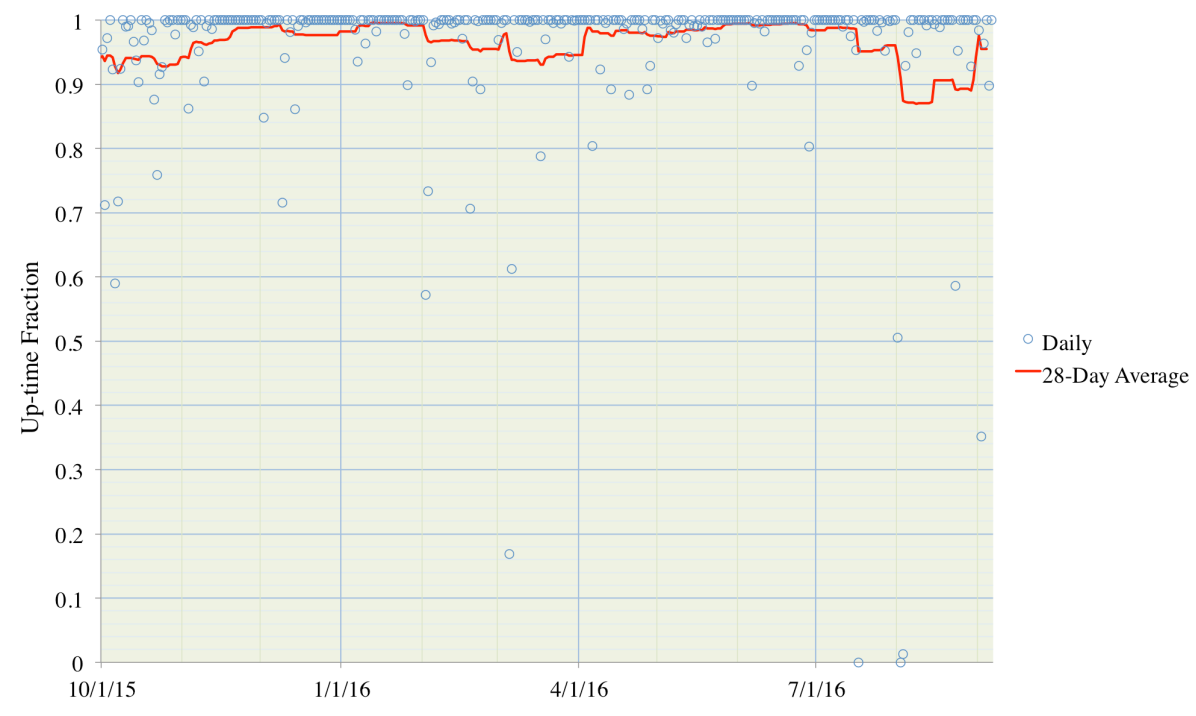

Figure N-3: The daily (points) and 28-day average (red line) NOvA near detector uptime for FY16. Overall the detector operated $>96 \%$ of the time during FY16, and recorded data for $99.1 \%$ of protons delivered to NuMI.

The near detector uptime was above $96 \%$, with $99.1 \%$ beam-weighted uptime. A single FEB and 11 APDs were swapped during FY 2016 through August, with another 66 planned for September. The vast majority of those were known to have had sub-optimal performance since soon after their installation, arising from a primer that was applied in production to the early batches of APDs. The usage rate of spare APDs has now been determined to be low enough to permit their use for the purpose of eliminating these lower-performing channels.

The NuMI beam was operated with the polarity of the magnetic horns set to produce a beam primarily of neutrinos ("Forward Horn Current, FHC") for most of FY 2016 beam running, with $4.1 \times 10^{20}$ POT delivered. For the last month of the FY $2016 \mathrm{NuMI}$ run the beam was configured for "Reverse Horn Current" (RHC) to deliver a beam consisting predominantly of anti-neutrinos, for an exposure of $0.66 \times 10^{20} \mathrm{POT}$. The near detector data will be used to tune simulation and reconstruction of anti-neutrino interactions in advance of a more substantial anti-neutrino run that will start in FY 2017. Figures N-1, N-2, and N-3 show the detector uptimes and delivery of protons to NuMI for FY 2016.

NOvA's first oscillation results based on an exposure of $2.74 \times 10^{20}$ POT (full-detector equivalent) were published in peer-reviewed journals, with the muon neutrino disappearance result presented in Phys. Rev. D 93, 051104 (R), and the electron neutrino appearance result in Phys. Rev. Lett. 116, 151806.

A second round of NOvA oscillation analysis results was announced at the Neutrino 2016 meeting in London in July by NOvA collaborator Prof. Tricia Vahle, and in a Fermilab Wine \& Cheese Seminar by Dr. Christopher Backhouse. The new results were based on the $6.05 \times 10^{20}$ POT (full-detector equivalent, accounting for data taken during construction of the far detector in FY 2014) accumulated during all NOvA running from February 2014 through May 2, 2016. Several improvements and updates to the analysis over that of the first results led to an increase in sensitivity beyond that expected from the increase in statistics alone. 
The largest systematic uncertainty in the first muon neutrino disappearance result was associated with a mismatch between the hadronic energy spectrum in simulated and observed muon neutrino candidate events in the near detector. In the first analysis, an ad hoc calibration shift was applied to achieve agreement in the mean hadronic energy between data and simulation, with the full size of the shift (14\%) taken as the systematic uncertainty on the hadronic energy scale. In the new analysis, this uncertainty per se was eliminated by including scattering from correlated nucleon pairs in the simulation of neutrino interactions as motivated by observations made in other recent neutrino experiments, including MINERvA. The available simulation of this channel in the GENIE neutrino interaction generator, the empirical Meson Exchange Current model, required ad hoc tuning to optimize the match to the NOvA data. Fig. N-4 shows the hadronic energy distribution with and without the MEC simulation.
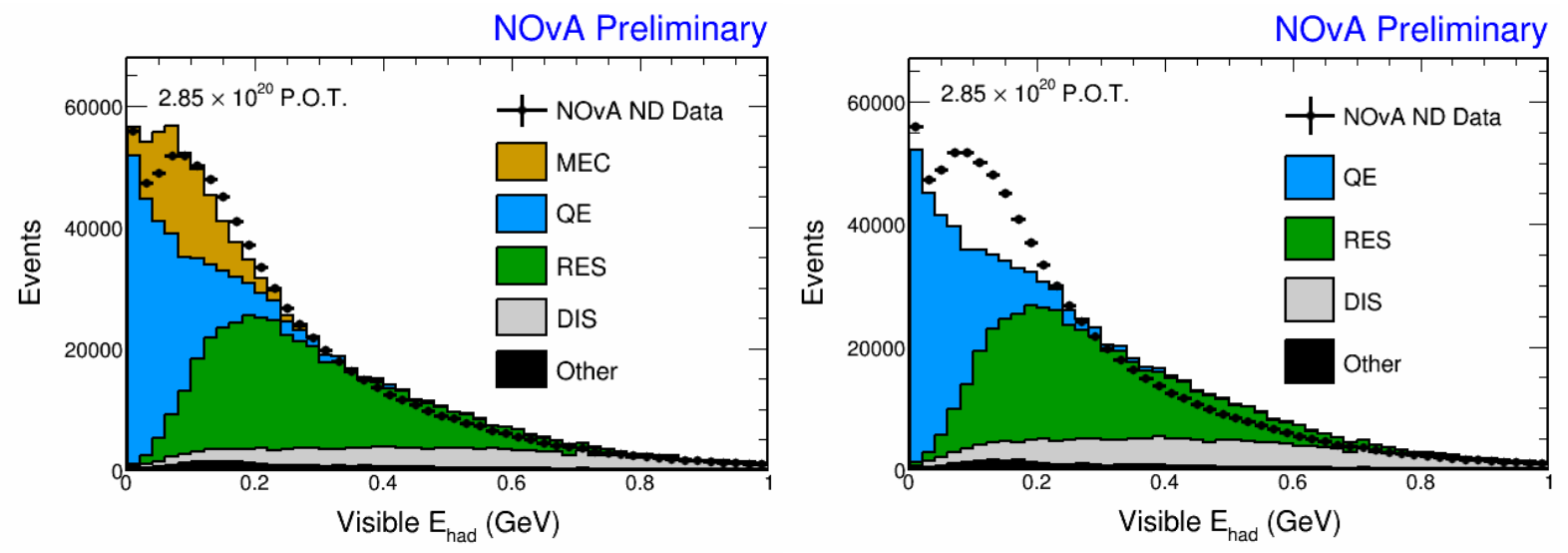

Figure N-4: The reconstructed hadronic energy spectrum in the NOvA near detector data (points), compared to Monte Carlo simulation. Left: default GENIE 10.2.4, without MEC channel. Right: GENIE 10.2.4, including the optional MEC model of neutrino scattering from correlated nucleon-pairs with additional tuning.

Figure N-5 shows the reconstructed muon neutrino energy spectrum recorded at the far detector, and the expectation in the absence of oscillations. 473 events are predicted in the absence of oscillations based on observations in the near detector, and 82 observed. The confidence intervals for the mass splitting $\Delta \mathrm{m}_{23}^{2}$ and the mixing angle $\theta_{23}$ resulting from a 3-flavor oscillation fit to the data are also shown in Fig. N-5. The result, while compatible with NOvA's first result and results of MINOS and T2K, excludes maximal mixing $\left(\sin ^{2}\left(\theta_{23}\right)=0.5\right)$ at 2.5 sigma, perhaps indicating a broken symmetry between the muon and tau flavor contributions in the neutrino sector. The chi-squared of the fit, 41.5 per 17 degrees of freedom, is large, but comes from the higher energy part of the spectrum that has little impact on the exclusion of maximal mixing.

The new electron neutrino appearance analysis incorporates several improvements. In general, selection criteria were optimized for measurement of the electron neutrino appearance probability, as opposed to its mere detection in the first analysis. Furthermore, the interpretation of the data was changed from a simple counting experiment to a fit of the oscillation probability to the data in bins of neutrino energy and selection purity. The largest single improvement was the development of a new deep-learning algorithm used to select electron neutrino events, the Convolutional Visual Network (CVN) based on recent developments in computer vision. CVN 
achieved signal selection efficiency approximately $40 \%$ greater than the two identifiers used in the first analysis, at a similar purity.
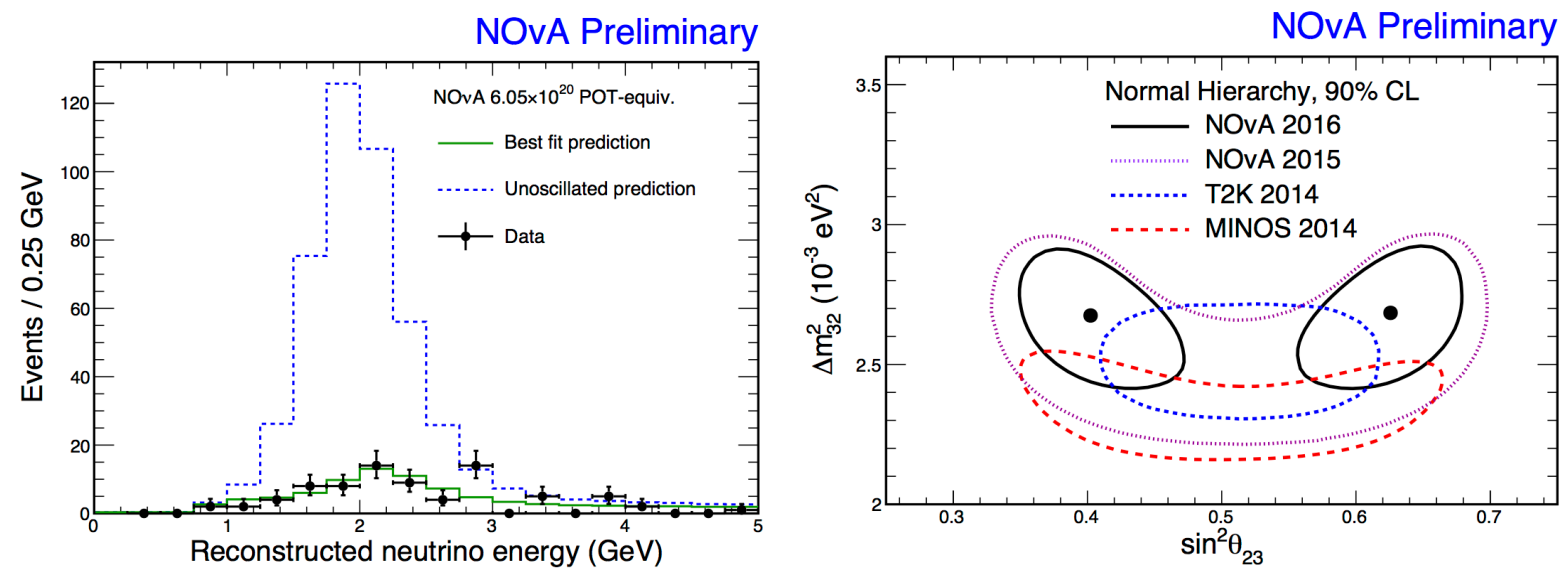

Figure N-5: Left: The reconstructed energy spectrum of muon neutrino candidate events in the far detector (points), with best oscillation fit (green line) and the prediction in the absence of oscillations (blue dashed line). Right: The resulting confidence intervals in $\Delta \mathrm{m}_{23}^{2}$ and $\sin 2\left(\theta_{23}\right)$ in black, compared to the early NOvA result and results of MINOS and T2K. There is no meaningful statistical preference between the two best fit points in the lower and upper octant of $\theta_{23}$.

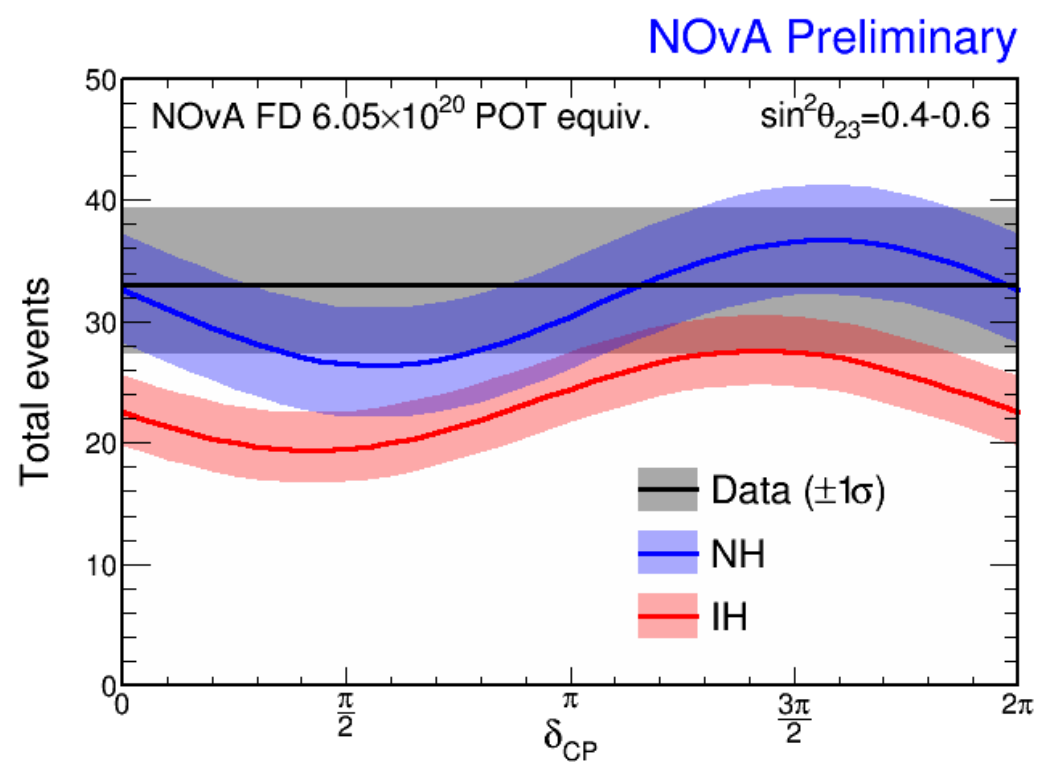

Figure N-6: Expected number of electron neutrino candidate events in the far detector, based on observations in the near detector, vs the major unknown parameters (red and blue bands), and the actual observation in the far detector (grey band).

When combined with the precise measurement of $\theta_{13}$ from reactor neutrino disappearance experiments and the NOvA muon neutrino disappearance result, the electron neutrino appearance result can be interpreted as constraints on the remaining unknowns. Fig. N-8 shows the preliminary resulting significance contours. The combined parameter region of $\delta_{\mathrm{CP}} \sim \pi / 2$, $\sin ^{2}\left(\theta_{23}\right)<0.5$ (i.e., the lower octant) and Inverted Mass Hierarchy is excluded at more than 3 sigma. Inclusion of more data will continue to reduce the allowed parameter space, with the 
upcoming antineutrino data having particular impact in helping to resolve degeneracies in the oscillation probabilities between the effects of $\theta_{23}$ octant, Mass Hierarchy, and $\delta_{\mathrm{CP}}$.

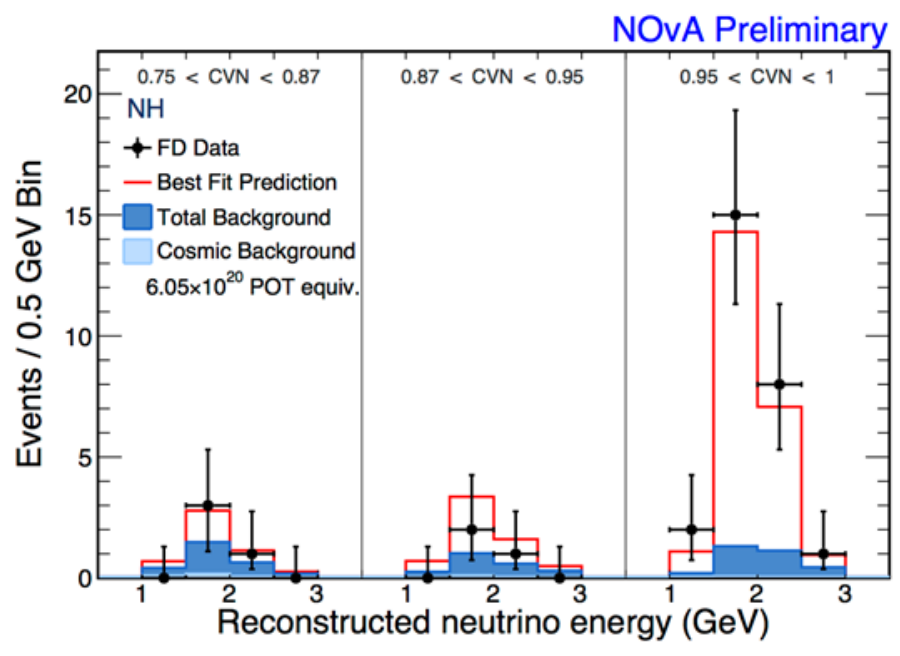

Figure N-7: The spectrum of observed electron neutrino candidate events in the far detector in bins of neutrino energy and CVN selector output value, compared to the best fit oscillation hypothesis assuming the Normal Mass Hierarchy. The total backgrounds are shown in blue.
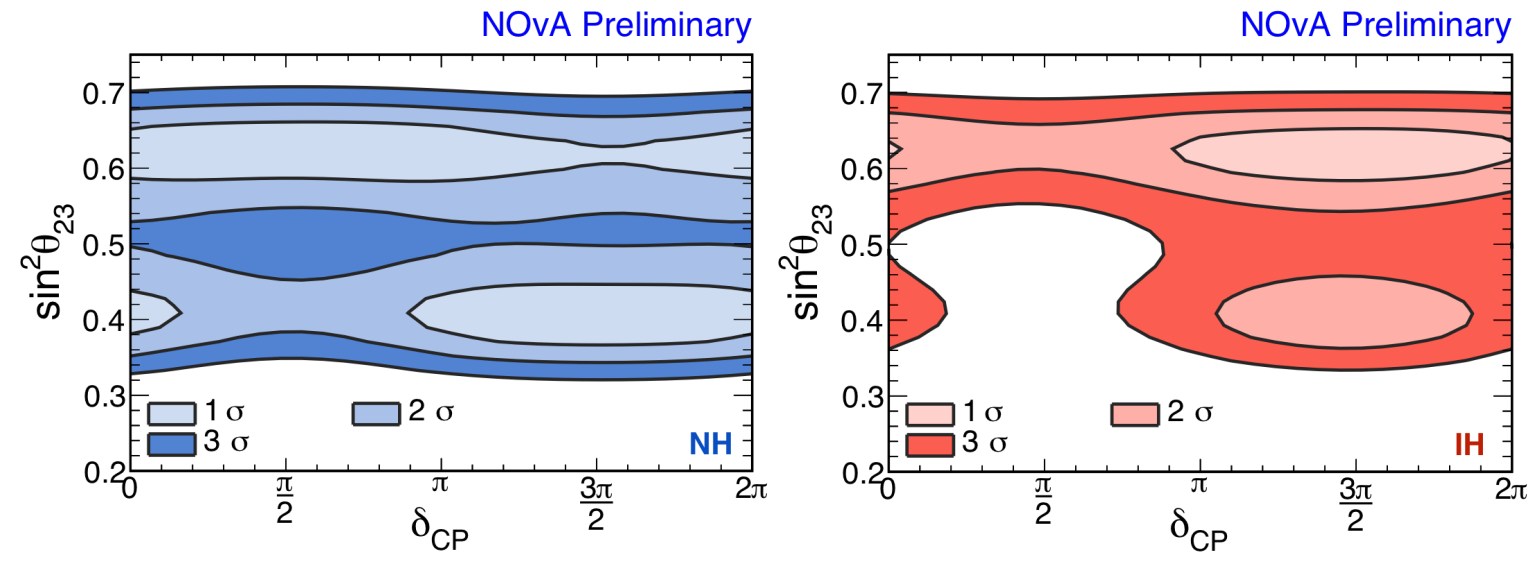

Figure N-8: The significance contours in $\sin ^{2}\left(\theta_{23}\right), \delta_{\mathrm{CP}}$, and the Mass Hierarchy (Left: Normal Hierarchy, Right: Inverted Hierarchy) for the new NOvA electron neutrino appearance result, when constrained by reactor neutrino disappearance measurements and the new NOvA muon neutrino disappearance result.

The result of NOvA's first search for sterile neutrino-induced oscillations were presented by Dr. Gavin Davies at a Fermilab Wine \& Cheese Seminar in July. The analysis compares the rate of neutral current interactions - which are not sensitive to oscillations among the 3 known flavors in the near and far detectors. Based on observation in the near detector, 84 candidate neutral current events, with a systematic uncertainty of $10 \%$, are expected in the far detector. Ninetyfive events are observed, leading to limits on the mixing angle $\theta_{34}$ that are competetive with that of the MINOS experiment. Future NOvA limits are expected to be competetive with those of the Super-Kamiokande experment. 
E-938 / MINERvA Report (D. Harris, K. McFarland)

The MINERvA experiment took physics quality data in in the NuMI Low Energy beam from November 2009 until summer 2013. In FY 2014 and FY 2015, the MINERvA experiment took data in the NuMI Medium Energy beam, and continued this data taking in FY 2016.

\section{FY 2016 Operations}

The data taken in FY 2016 was mostly Medium Energy neutrino data (6 GeV peak neutrino energy) with roughly four weeks of antineutrino data taken just before the Summer 2016 shutdown. Between October 1, 2015 and September 30, 2016, the experiment received 4.10 $\times 10^{20}$ POT in neutrino mode, and $0.66 \times 10^{20}$ POT in antineutrino mode. Since 2013 the experiment has received $11.1 \times 10^{20}$ POT in medium energy running, taken with an integrated livetime of $95.9 \%$.

During the FY 2016 running period, the collaboration continued to calibrate the detector, monitor the detector light levels, and check the reconstruction performance of both the MINERvA and MINOS detectors continuously. The experiment operates $40 \%$ of its shifts remotely, and in FY 2016 has started check-listed based owl shifts with no significant increase in detector downtime thanks to new automated texting capabilities in case of DAQ outages. Figure MV-1 shows the FY 2016 protons delivered and recorded versus time, including the POT recorded when the MINERvA detector was live and, noting that some MINERvA analyses use the MINOS near detector, the POT recorded when both MINERvA and the MINOS Near Detector were live. In FY 2016, MINERvA took data with no helium in the cryostat in order to do an empty target subtraction, but did fill its water target on February 22, 2016 and proceeded to take $2.5(0.66) \times 10^{20}$ POT in neutrino (antineutrino) mode on the full water target.

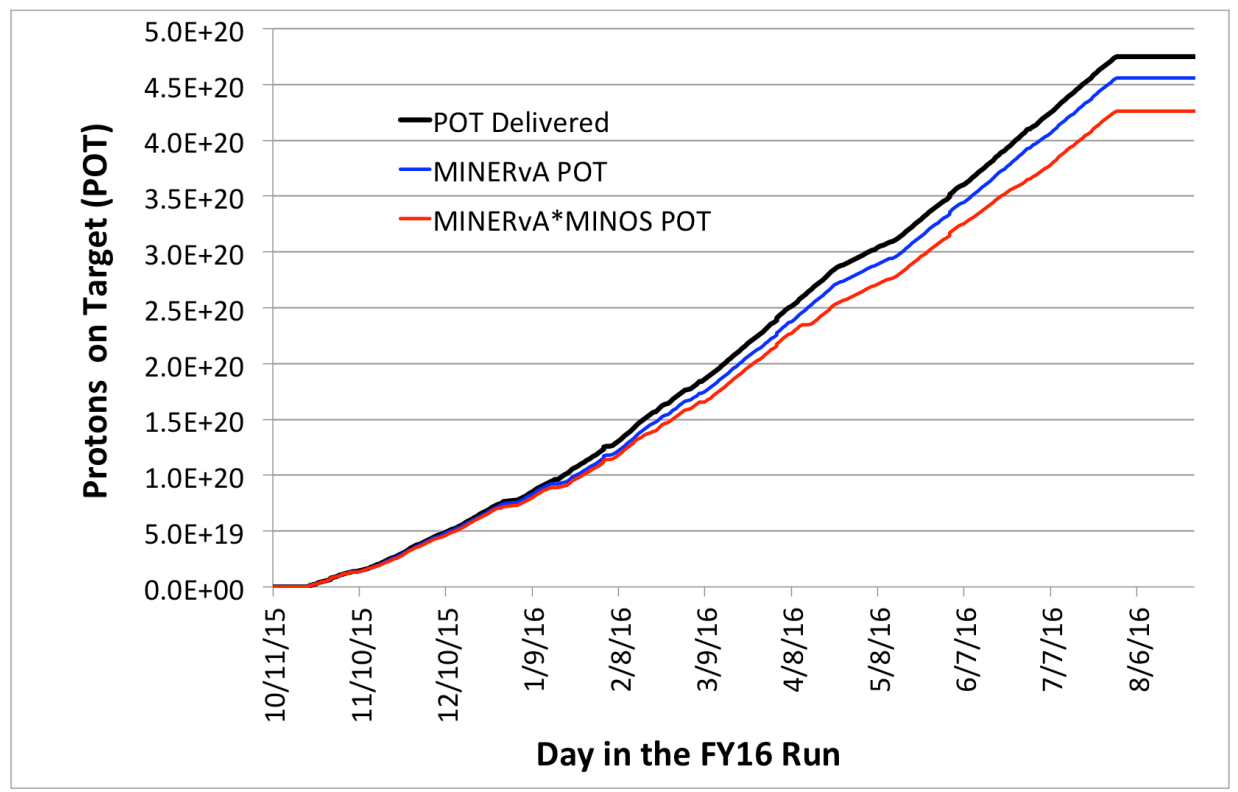

Figure MV-1: Shows the protons on target versus time, as well as the integrated livetimes of the MINERvA detector alone and the MINERvA and MINOS Near detector combined. 
MINERvA Construction and Installation Activities in FY 2016

Over the course of FY 2016, MINERvA collaborators together with PPD's EED group, continued to upgrade the data acquisition firmware so that the experiment would have a reduced dead time associated with each time a readout cycle was initiated, at the expense of recording the lowest gain channels for every readout cycle. In the new scheme the low gain channels, which are used only rarely, are read out only one time after the spill. Another issue found in the current firmware that will be corrected in the new firmware is associated with the corruption of data for spills where a front-end board had more than 21 readout events. This newest firmware version (v97) is being installed in the underground neutrino detector during the FY 2016 shutdown.

\section{MINERvA Results in FY 2016}

The low-energy neutrino data, which is still being analyzed, was taken to provide exclusive cross-section measurements on a variety of nuclei. Low energy events tend to have few final state particles - which allows the identification of single particles in the MINERvA detector and the measurement of exclusive channels important for current and future oscillation experiments. The MINERvA detector has a granularity that is about a factor of 10 more than the NOvA detector, and can be used to identify processes that will contribute backgrounds to NOvA. MINERvA sees a higher neutrino beam energy than T2K's near detector, and measures reactions that contribute backgrounds to T2K from the high-energy tail (above $1 \mathrm{GeV}$ ) of the beam.

By the start of 2016, the MINERvA Collaboration had published a total of 8 papers covering a broad range of neutrino and antineutrino interaction channels including: different ways of analyzing the charged current quasi-elastic interaction; a measurement of the inclusive charged current cross section ratios lead/scintillator, iron/scintillator, and carbon//scintillator; both neutrino and antineutrino production of charged and neutral pions; coherent production of charged pions by both neutrinos and antineutrinos; and a first electron neutrino charged current quasi-elastic cross section measurement.

In 2016 the MINERvA Collaboration published an additional 8 papers on neutrino interactions in the Low Energy Beam configuration, and has submitted a publication describing the most precise a priori neutrino flux prediction for the NuMI beamline. These publications include two new measurements of channels that have never been seen before (coherent kaon production [Phys. Rev. Lett. 117, 061802 (2016)] and diffractive neutral pion production, [arXiv: 1604.01728 [hepex] to appear in Phys. Rev. Lett.]. MINERvA has also published several new differential cross sections to better characterize both neutral and charged pion production by antineutrinos and neutrinos, kinematic variables that are important for modeling pion production (arXiv: 1606.07127[hep-ex] to appear in Phys. Rev. D), and an increase of the statistical precision of the world's charged current kaon production sample by a factor of 20 [Phys. Rev. D 94, 012002 (2016)]. The Collaboration also published in 2016 the Deep Inelastic Scattering cross section ratios for lead, iron, and carbon to scintillator to directly search for partonic nuclear effects in neutrino scattering [Phys. Rev. D 93, 071101 (2016)].

One FY 2016 result has already had a large impact on current neutrino oscillation experiments, namely the measurement of nuclear effects in neutrino-carbon interactions at low threemomentum transfer. Until this publication, measurements of quasi-elastic-like neutrino interactions were either of the leptonic side or of the hadronic side (looking only at the extra 
energy near the interaction vertex, or the proton momentum distribution in quasi-elastic events). By looking for the first time at the visible recoil energy in the event as a function of the momentum transferred to the nucleus, MINERvA was able to identify two different predicted nuclear effects: (i) an effective screening of the nucleus which occurs at the lowest recoil and momentum transfer ("Random Phase Approximation", or RPA), and (ii) an effect associated with nucleon-nucleon correlations inside the carbon nucleus, where the cross section "dip" between the quasi-elastic and resonance peaks is filled in with events.

These measurements show that the Nieves model of nucleon correlations (arXiv: 1601.02038), which was not originally in neutrino event generators, partially helps resolve the discrepancy between the data and the predictions. However, the data show greater enhancement in this dip region than even the Nieves model predicts, and this will be incorporated into future neutrino event generators for accurate predictions of visible neutrino energy. NOvA has adopted a very similar analysis because of a similar discrepancy in its near detector hadron energy distribution, and because of this improvement in their model of neutrino interactions, NOvA was able to reduce their largest systematic uncertainty, that of their hadronic energy scale, from $14 \%$ to $5 \%$. $\mathrm{T} 2 \mathrm{~K}$ has also made direct use of these results in their oscillation analysis, in addition to the MINERvA FY 2015 results on coherent pion scattering.

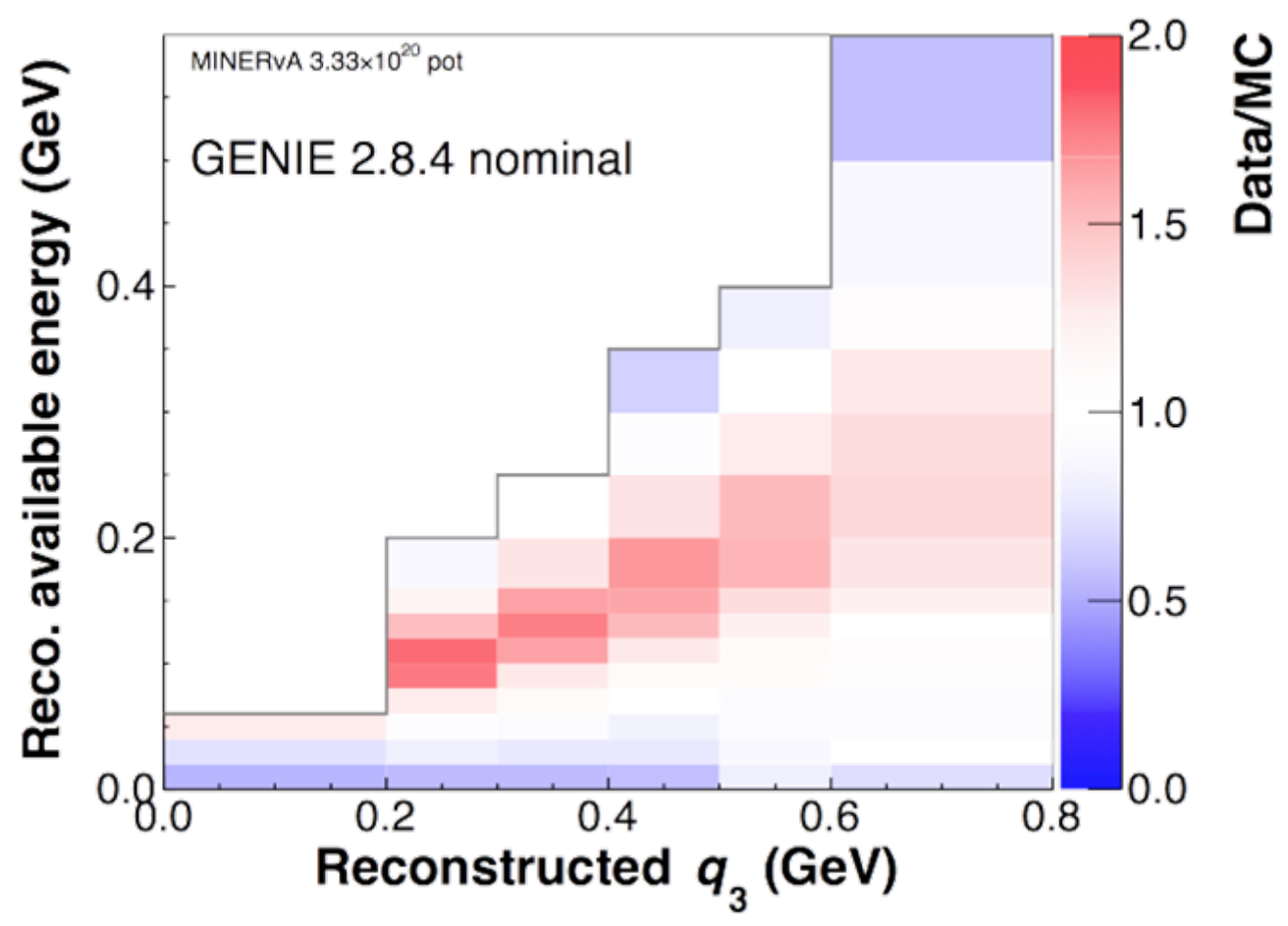

Figure MV-2: Shows the ratio of data to prediction for charged current $v_{\mu}$ events at low momentum transfer $\left(\mathrm{q}_{3}\right)$. Using the nominal event generator used for MINERvA, NOvA, and T2K's near detector. There are two effects seen at high statistical significance: one is a suppression of the cross section at low available energy, and the other is an increase in cross section in the dip between the quasi-elastic and delta production 


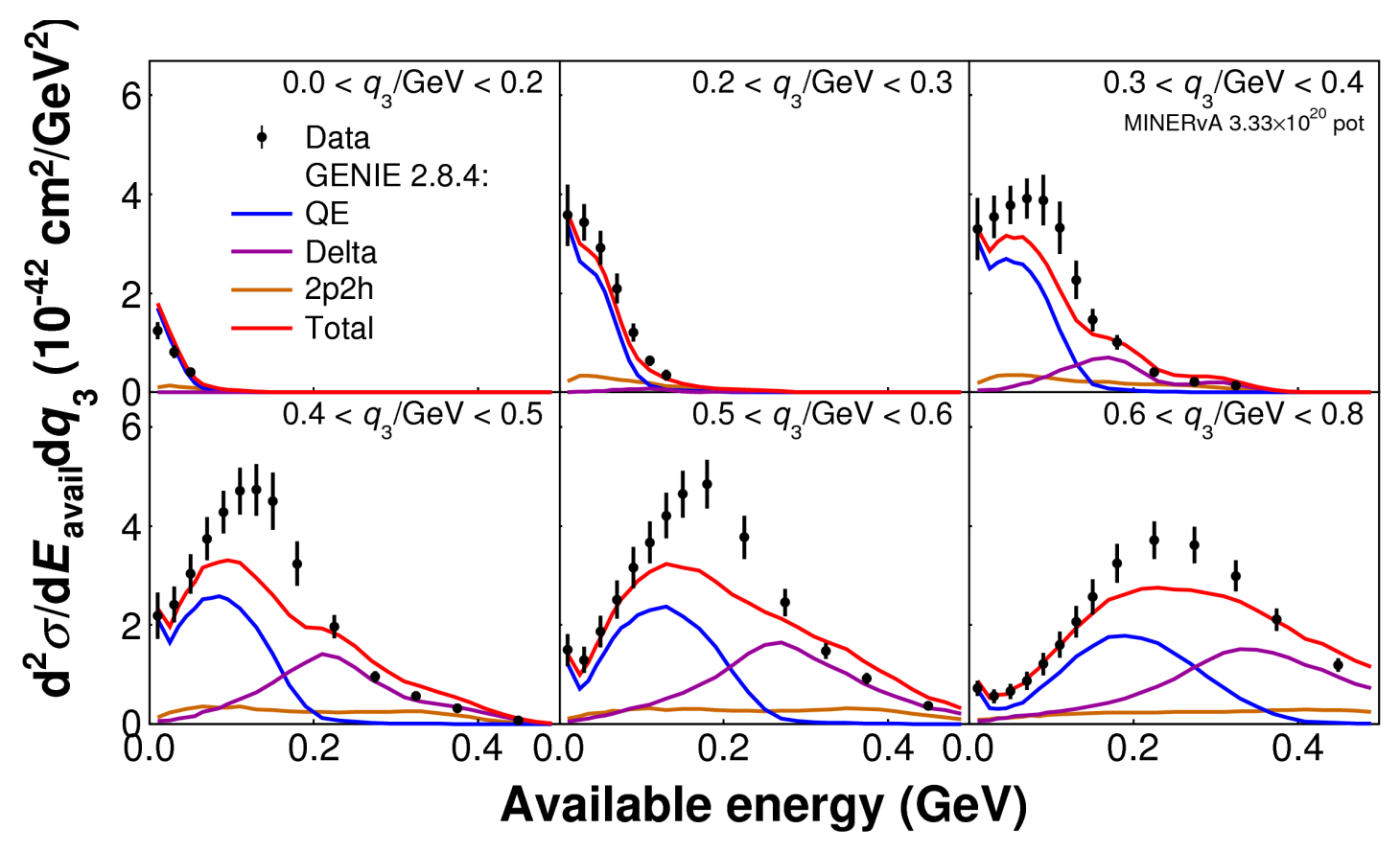

Figure MV-3: Shows a differential cross sections as a function of available energy for different 3-momentum transfer bins, for both the data, the nominal neutrino event generator prediction, and after including the change in the predicted cross section with both RPA and multi-nucleon correlation effects added.

MINERvA has also recently provided several tools that will be useful to oscillation experiments for flux predictions For example, MINERvA's FY 2016 publication on neutrino electron elastic scattering (Phys. Rev. D 93, 112007) is an important proof of principle for how DUNE can measure its neutrino flux very precisely, without systematic uncertainties from nuclear effects in neutrino interactions. The scattering of neutrinos off atomic electrons is the one neutrino interaction whose cross section can be reliably calculated with a precision of better than one per cent. The experimental challenge in using these interactions for an in situ flux measurement is that the cross section is 2000 times smaller than neutrino-nucleus interactions, and so the event rate is small and the backgrounds can be high. Because of the good angular resolution of MINERvA, and the ability to separate electron showers from photon showers, the experiment collected 114 neutrino electron scattering events after background subtraction, and used that event rate and the electron energy spectrum to put a constraint on the flux prediction whose uncertainty is similar in size to the uncertainties in the a priori flux prediction. This technique will also be particularly powerful for MINERvA in the medium energy beam where the number of neutrino electron scattering events in neutrino mode alone is already expected to be a factor of 10 higher based on the higher flux, higher integrated POT in the Medium Energy configuration, and the higher neutrino electron scattering cross section because of the higher neutrino beam energy. 

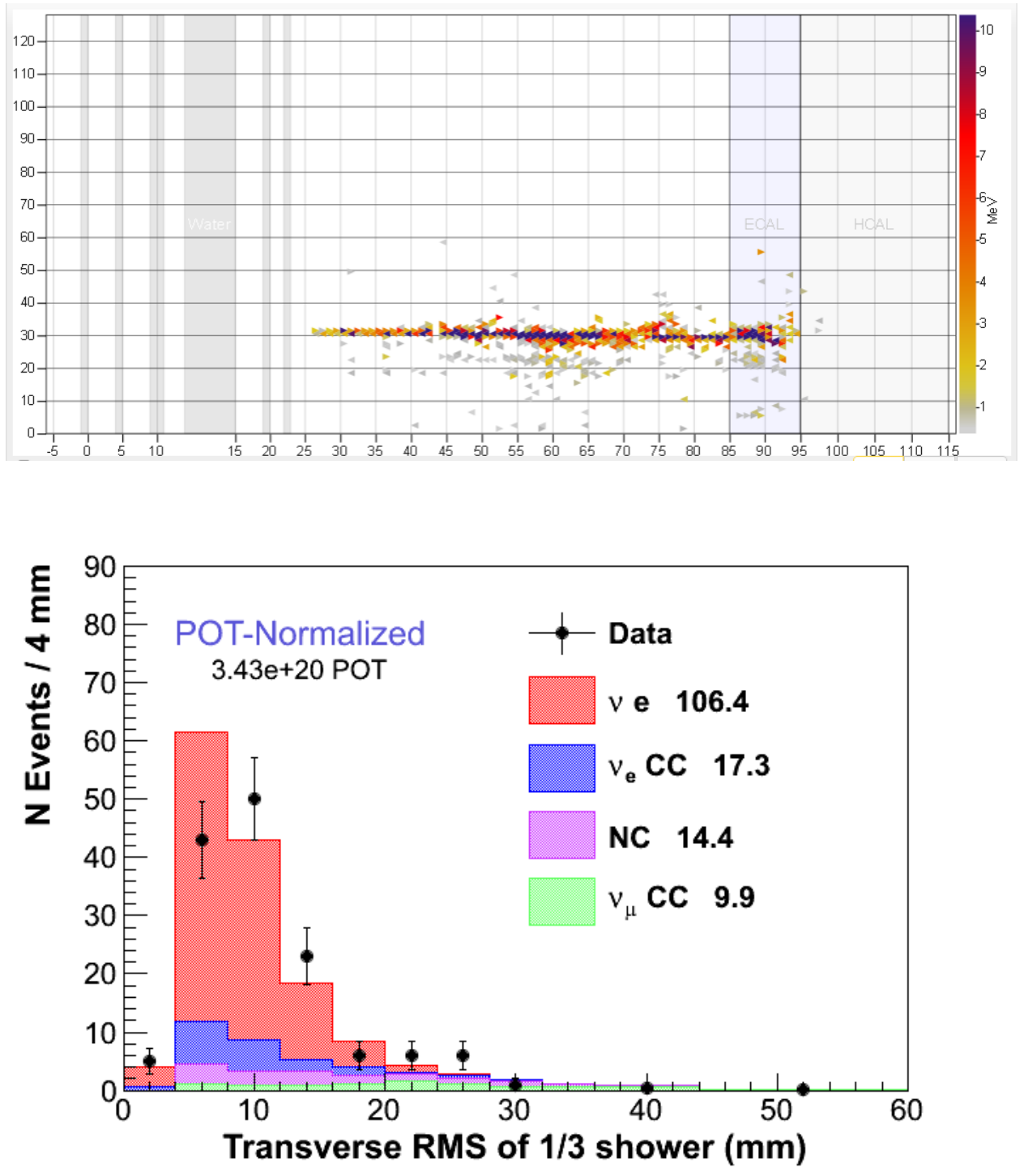

Figure MV-4 Top: An event display from the data showing a candidate for a neutrino scattering off an atomic electron in the detector. Bottom: The electron energy times the square of the angle of the electron with respect to the neutrino beam: the signature of neutrino electron scattering events is that this quantity is related to the electron mass

Fixed Target Switchyard 120 GeV (SY120) and MTest Reports (D. Geesaman, C. D. Moore, P. E. Reimer, M. Rominsky, J.J. Schmidt)

The general operation of SY120 was relatively smooth. The beam for SeaQuest was operated with the same intensity as last year, as requested by the experiment, and with the maximum achievable duty factor, and manageable beam losses. The MTest beamline was operated for the FTBF program, as requested by the test beam experiments. The MCenter Beamline with a tertiary beam capability was operated as needed for the LArIAT experiment. Instrumentation improvements continued to be developed with the main emphasis on improving the BPM system. During the FY 2016 shutdown work continued on cable remediation. 
E906/SeaQuest Drell-Yan (D. Geesaman, P. E. Reimer)

The SeaQuest experiment (E906) is measuring the ratio of the d-antiquarks to u-antiquarks in the proton and the nuclear dependence of the antiquark distributions in the $\mathrm{x}_{\mathrm{Bj}}$ range of 0.1-0.5 by measuring the relative rates of proton-induced di-muon production (Drell-Yan production) on targets of ${ }^{1} \mathrm{H},{ }^{2} \mathrm{H}, \mathrm{C}, \mathrm{Fe}$ and W. Additional measurements from the same data set will include the energy loss of fast quarks in cold nuclear matter, decay angular distributions, the nuclear dependence of $\mathrm{J} /$ Psi production and a search for dark photons.

SeaQuest has been accumulating production data since the spring of 2014. One issue that complicates the accounting of beam delivery is the instantaneous duty factor of the extracted beam. RF buckets containing significantly more protons than the average (factors of 5-50) greatly increase the rate of random triggers and increase the complexity of event reconstruction, leading to a decrease in the reconstruction efficiency. To deal with these issues, the experiment uses a beam Cerenkov counter to veto events for a period of $190 \mathrm{~ns}$ around beam buckets with greater than 5-10 times the normal intensity. Through October 2015, the accelerator delivered $1.8 \times 10^{18}$ protons to the experiment but only $1.1 \times 10^{18}$ protons survived this beam veto cut and $0.85 \times 10^{18}$ were recorded due to the apparatus and data acquisition dead time (Fig. SQ-1).

During the summer 2015 shutdown, the experiment installed a new tracking chamber with larger acceptance and better rate handling capabilities in front of the analysis magnet, replacing a 20year-old chamber. Unfortunately, this new chamber experienced a number of broken wires in early operation. Due to the flammable chamber gas in use, while chamber removal, repair, and reinstallation would only take 2-3 days, flushing and refilling the gas consumed about 7 days for each repair. In March 2017, we changed to a configuration with both the old and new chambers in place, (though only the outer portions of the new chamber providing the increase in acceptance were instrumented) and ran successfully through the end of July with no additional chamber failures. By the end of July 2016, the total number of live protons was $1.2 \times 10^{18}$. The duty factor remained at the 40-45\% level maintained in the summer of 2015.

SeaQuest currently has 9 graduate students. Five students have completed their Ph.D's on the experiment. Typically 7 post-doc have active roles in the experiment and 10 undergraduates are involved each year.

During the summer shutdown in FY 2016, the experiment is updating the data acquisition to significantly reduce the readout dead time by a factor of 5-10. Electronics are being fabricated to fully instrument the new wire chamber. New trigger hodoscopes are under investigation to increase the efficiency of the trigger for dark photons.

Production analysis of all the data taken to date is almost complete. Preliminary results based on the data taken through FY 2015 have been presented at conferences and are shown for the ratio of d-antiquarks to u-antiquarks (Fig. SQ-2) in the proton and the nuclear dependence of the cross sections (Fig. SQ-3). While agreeing well with previous E886 results at low x, these results differ considerably from the much lower statistics data at high $\mathrm{x}$ from E866 and indicate that the enhancement of $d$-antiquarks persists at $x$ values above 0.25 . The result has significant implications for the origin of the non-perturbative sea of the proton and also has consequences for the LHC, favoring the relative cross section for producing $\mathrm{W}^{\prime}$ bosons at high mass (in $\mathrm{u}$ d- 
antiquark interactions) compared to that for $\mathrm{Z}$ ' bosons (in $\mathrm{u} \mathrm{u}$-antiquark interactions). There remains little room for substantial nuclear dependence of the cross sections. This observation constrains one of the systematic uncertainties in the nuclear corrections for deuterium used to extract the d-antiquark to u-antiquark ratio. It is expected that publications based on the data through FY 2016 will be submitted this fall.

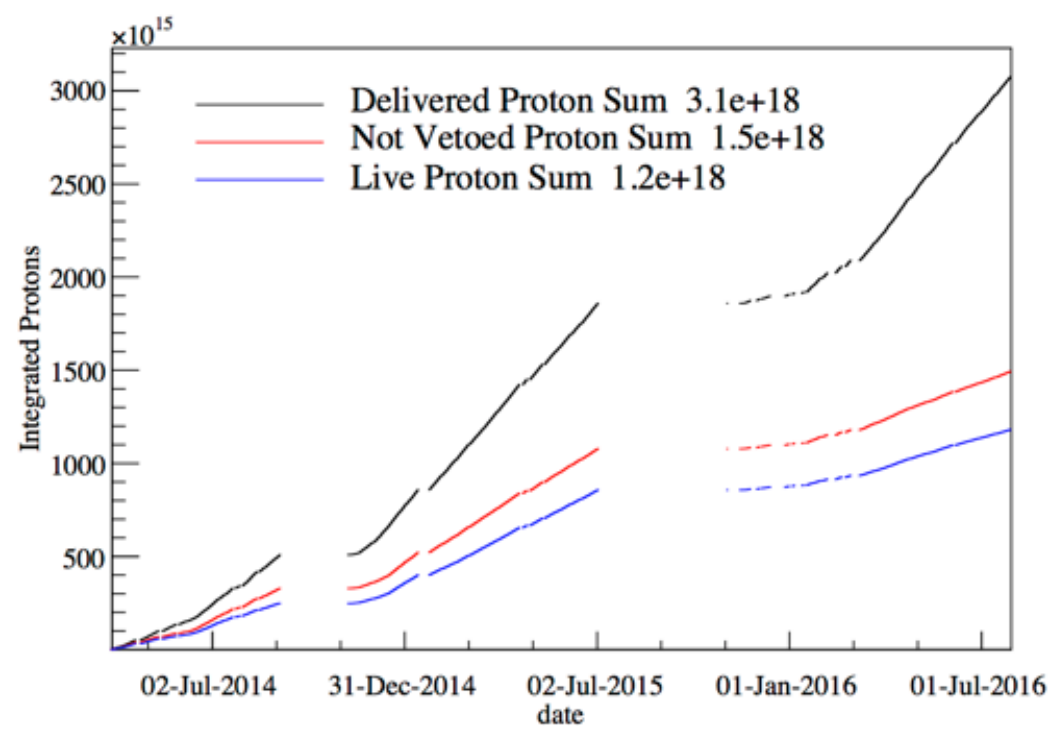

Figure SQ-1: The integrated protons delivered to SeaQuest is show in the top (black) line as a function of time. Of those protons the center (red) line indicates those protons delivered with acceptable instantaneous duty factor.

Finally the lower (black) line shows the effect of experimental dead time on integrated protons.

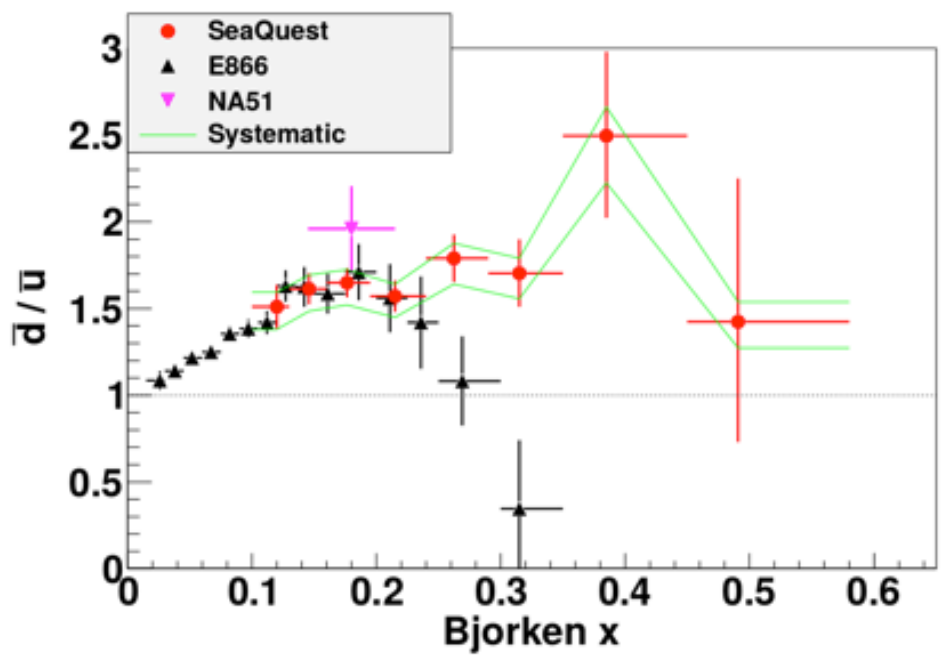

Figure SQ-2: The red points show the ratio of anti-d to anti-u quarks in the proton as a function of $\mathrm{x}$ extracted from data recorded through fall of 2015. The black squared show the same quantity previously measured in by Fermilab E866. 

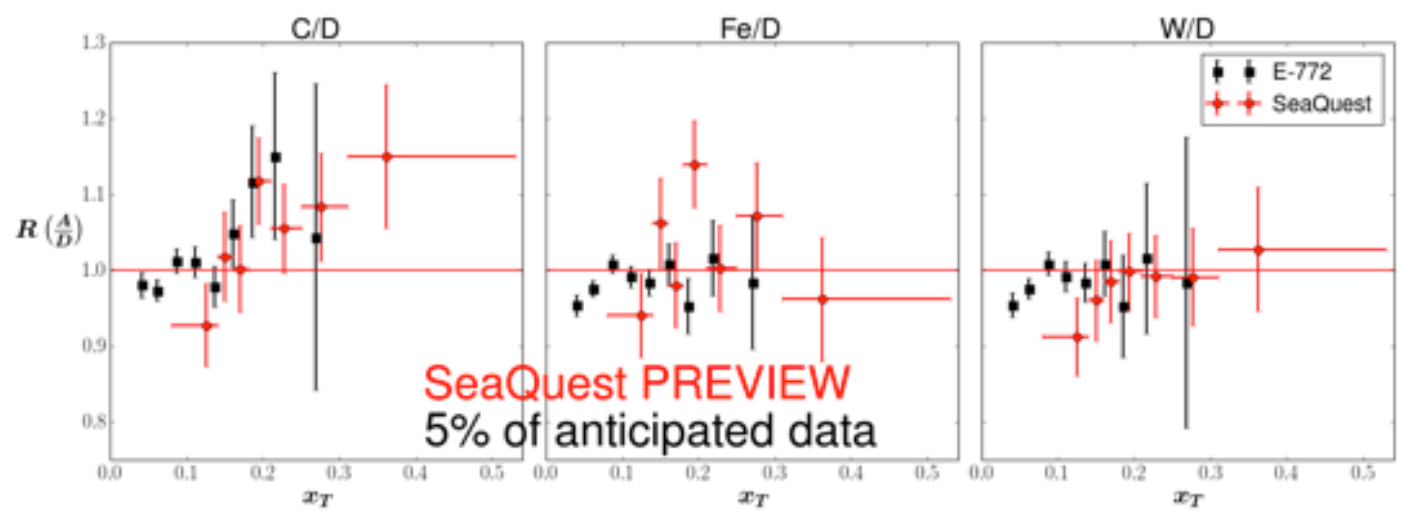

Figure SQ-3: The red points show the ratio of Drell-Yan cross sections for $\mathrm{C} /{ }^{2} \mathrm{H}$ (left), $\mathrm{Fe} /{ }^{2} \mathrm{H}$ (middle) and $\mathrm{W} /{ }^{2} \mathrm{H}$ (right) extracted from SeaQuest data recorded through fall 2015. The black squares represent previously measured Fermilab E772 data.

\section{The Fermilab Test Beam Facility (M. Rominsky, J.J. Schmidt)}

The Fermilab Test Beam Facility (FTBF) provides users from around the world an opportunity to test detectors in a variety of charged particle beams. The facility offers two beamlines (MTest and MCenter) to accomplish this goal. A plan view of the facility is shown in Fig. TB-1. The facility offers instrumentation to understand the beamline and infrastructure such as gas lines, high voltage lines, and signal cables. In FY 2016, results from test beam experiments were shown in 9 talks or posters at conferences. There are 10 publications and four articles in preparation.

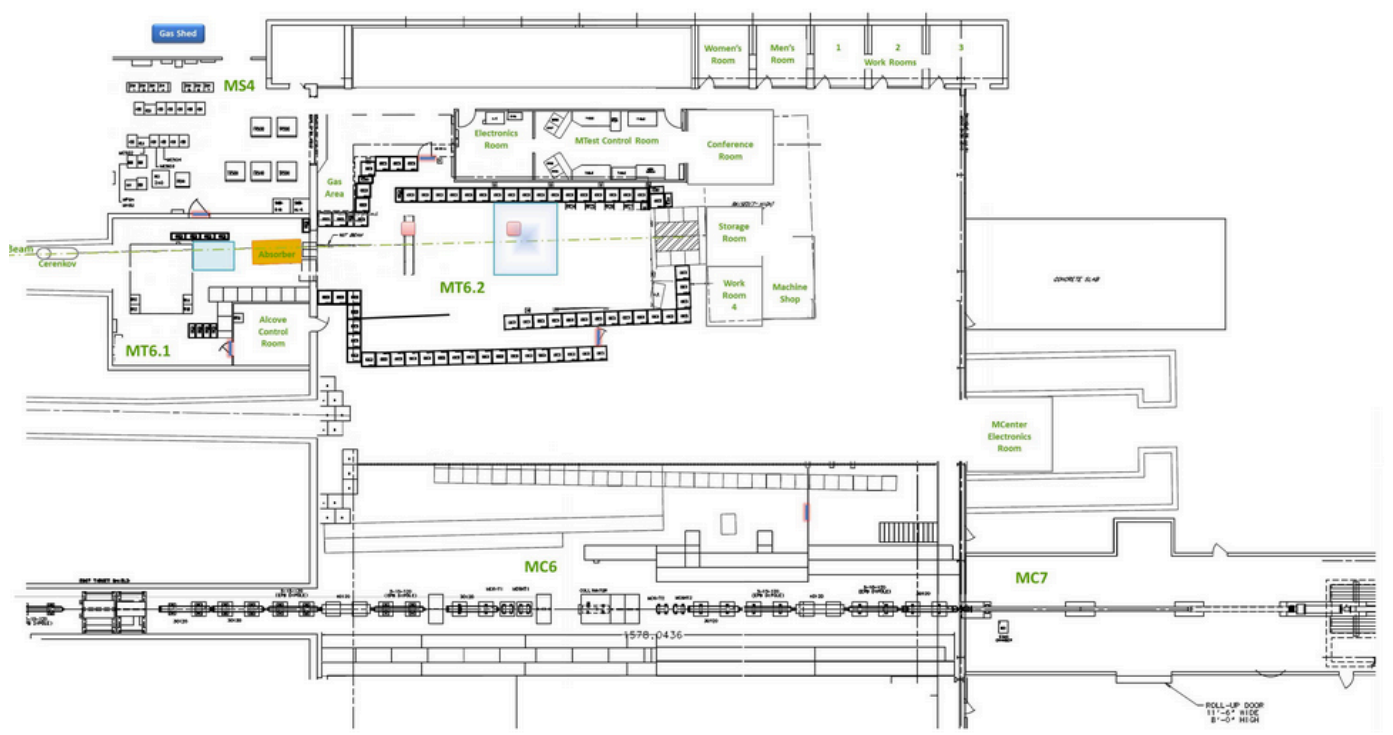

Figure TB-1: View of the Fermilab Test Beam Facility. The beamline at the top of the picture is MTest and the beamline at the bottom of the picture is MCenter. . 


\begin{tabular}{|c|c|}
\hline $\begin{array}{l}\text { Experiment } \\
\text { Number }\end{array}$ & Description \\
\hline T1041 & CMS Forward Calorimetry R\&D \\
\hline T0992 & Radiation Hard Sensors for the HL-LHC \\
\hline T1015 & Dual Readout Calorimetry with Glasses \\
\hline T1065 & Secondary Emitters Study \\
\hline T1068 & Beam Tests of the SVX4 Telescope \\
\hline T1043 & Mu2e Cosmic Ray Veto Detector \\
\hline T1044 & sPHENIX Calorimetry Tests \\
\hline T1048 & EIC PID \\
\hline T1037 & FLYSUB Consortium Tracking \& RICH \\
\hline T1018 & UCLA Spacordion Tungsten Powder Calorimeter \\
\hline T1042 & g-2 Straw Tracker Vacuum Tests \\
\hline T1073 & Precision Timing Profile Monitor \\
\hline T1072 & Muon Strips for Future Colliders \\
\hline T0979 & Fast Timing Counters for PSEC \\
\hline T1075 & Large Area ToF for ProtoDUNE \\
\hline T1034 & LArIAT: Liquid Argon in a Test Beam \\
\hline
\end{tabular}

Table TB-1: Test Beam experiments performed in FY 2016.

Research Performed at the FTBF in FY 2016

Each test beam experiment is required to prepare a Technical Scope of Work (TSW) with the laboratory in which the beam, infrastructure, and safety requirements are spelled out in detail. Four new experiments were approved in FY 2016 and started taking data. Twelve experiments returned to continue their programs. These 16 experiments are listed in Table TB-1 and represent 191 collaborators from 67 institutions in 13 countries. Figures TB-2 and TB-3 show how the experiments broke down by user classification and research focus. We supported groups from both ATLAS and CMS, as well as groups from Brookhaven and the Relativistic Heavy Ion Collider (RHIC). The Mu2e experiment tested a variety of their detectors and are now continuing 
to use the facility to develop their data acquisition system. The g-2 experiment used the facility to conduct rate of rise tests for their straw-tube detectors. We supported the neutrino experiments LArIAT and LAToF for ProtoDUNE. We also had several groups come in to test general detector research and development. Full information from these groups will be included in the annual test beam report (in preparation).

\section{User Breakdown by Classification}

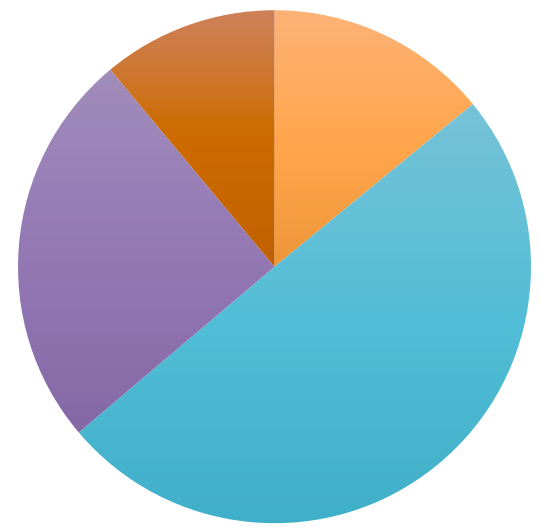

Postdoc Scientist/Faculty $\quad$ Student Engineer/Technician

Figure TB-2: Number of users broken down by classification. The total number of users for FY16 is 199 . Students include undergraduate and graduate students. Engineers and Technicians refer to people that experiments brought with them.

\section{Experiments by Research Focus}

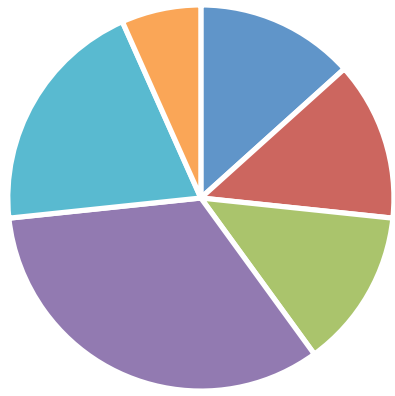

$$
\begin{array}{lll}
\text { - Neutrino } & \text { Muon } & \text { " Collider (LHC) } \\
\text { - Collider (Non LHC) } & =\text { General R\&D } & \text { - Outreach/Facility }
\end{array}
$$

Figure TB-3: The research focus of the different groups that came to the test beam in FY2016. We support a broad range of users. 
The experiments fully utilized the MTest beamline for data taking purposes out of the 35 weeks with beam available during the year. Many weeks included multiple users. Counting each user per week yields 51 weeks of experiment time during FY 2016. The MCenter beamline was used for data taking for the LArIAT experiment (T1034) and for the Large Area Time of Flight for ProtoDUNE (T1075). LArIAT ran well and is proposing a third run in FY 2017.

\section{Test Beam Publications}

1. Test Beam Studies of Silicon Timing for Use in Calorimetry. A. Apresyan, G. Bolla, A. Bornheim, H. Kim, S. Los, C. Pena, E. Ramberg, A. Ronzhin, M. Spiropulu, and S. Xie. NIM, A825 (2016) 62-68.

2. High time resolution photo-detectors for PET applications, Anatoly Ronzhin, invited by NIM editor article, Special Medical Edition. NIM, A809 (2016), 53-57.

3. Direct tests of micro channel plates as the active element of a new shower maximum detector, A. Ronzhin, S. Los, E. Ramberg, A. Apresyan, S. Xie, M. Spiropulu, H. Kim. NIM, A795 (2015), 52-57.

4. Study of the timing performance of micro channel plate photomultiplier for use as an active layer in a shower maximum detector. A. Ronzhin, S. Los, E. Ramberg, A. Apresyan, S. Xie, M. Spiropulu, H. Kim, NIM, A795 (2015), 288-292.

5. A feasibility study of a PET/MRI insert detector using strip-line and waveform sampling data acquisition, H. Kim, C.-T. Chen, N. Eclov, A. Ronzhin, P. Murat, E. Ramberg, S. Los, Alice M Wyrwicz, Limin Li, C.-M. Kao, NIM, A784 (2015), 557-564.

6. On Timing Properties of LYSO-based Calorimeters, D. Anderson, A. Apresyan, A. Bornheim, J. Duarte, C. Pena, A. Ronzhin, M. Spiropulu, J. Trevor, S. Xie, NIM, A794 (2015) 7-14.

7. Precision timing measurements for high energy photons, Dustin Anderson, Artur Apreysan, Adi Bornheim, Javier Duarte, Harvey Newman, Cristian Pena, Anatoly Ronzhin, Maria Spiropulu, Jason Trevor, Si Xie, Ren-Yuan Zhu, NIM, A787 (2015) 94-97.

8. Development of a new fast shower maximum detector based on microchannel plate photomultipliers (MCP-PMT) as an active elements, A. Ronzhin, S. Los, E. Ramberg, A. Apresyan, S. Xie, M. Spiropulu, H. Kim, A. Zatserklyaniy, NIM, A759 (2014), 63-73.

9. Status of ADRIANO R\&D in T1015 Collaboration, C. Gatto et al. 2015 J. Phys.: Conf. Ser. 587012060 .

10. Preliminary Results from a Test Beam of ADRIANO Prototype, C. Gatto et al. 2012 J. Phys.: Conf. Ser. 404012030

\section{ANNIE Experiment in the SciBooNE Hall (M. Sanchez, M. Wetstein)}

Phase I of the Accelerator Neutrino Neutron Interaction Experiment (ANNIE) was approved, installed, commissioned, and operated during FY 2016. ANNIE is a 30-ton Gadolinium-loaded 
Water Cherenkov detector, situated on the Booster Neutrino Beam and designed to measure final state neutron production from neutrino interactions. ANNIE is also designed to serve as a first working demonstration of Large Area Picosecond Photodetectors (LAPPDs) in a physics measurement.

ANNIE Phase I consisted of the full ANNIE tank, instrumented with a small number of conventional phototubes. The goal of this phase is to measure background neutrons originating from beam neutrino interactions on the target and dirt upstream of the experiment. The left and right panels of Fig. AN-1 show the installation and water fill of the ANNIE detector.

Commissioning of the detector was completed by April of 2016, and the collaboration collected roughly 3 months of BNB data, now being analyzed. Figure AN-2 shows the first observation of beam neutrinos. ANNIE Phase IB, an extension of the current operation, is approved and funded for FY 2017. This phase, which requires no major modifications to the current setup, will allow the collaboration to finish testing the systems needed for the main physics measurement.

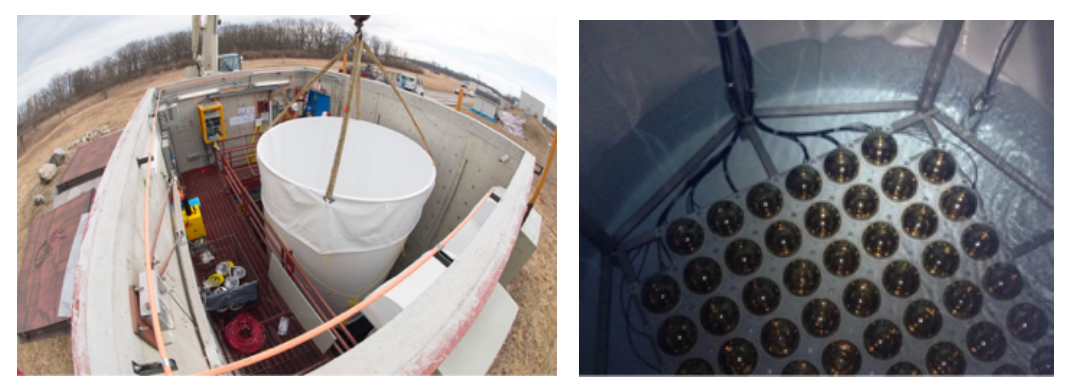

Figure AN-1: Left: The ANNIE water tank being lowered into the experimental hall (formerly SciBooNE). Right: A picture inside the tank, as it was being filled with ultra-pure water.

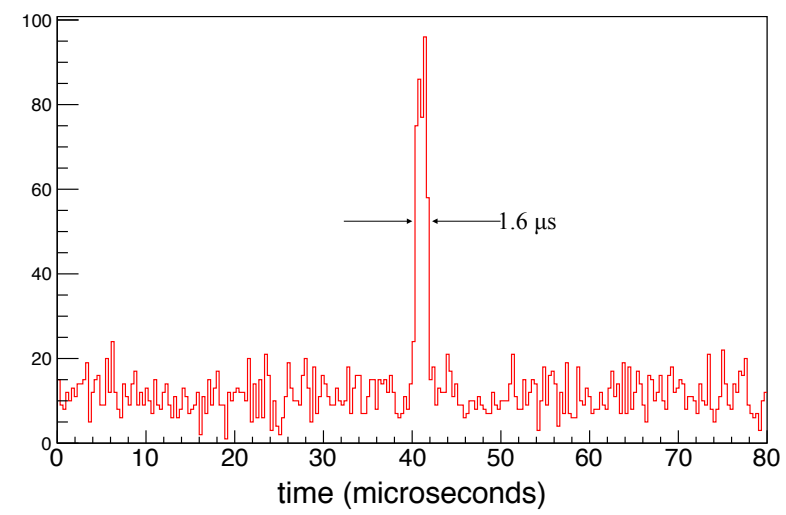

Figure AN-2: First observation of beam neutrinos.

\section{Acknowledgement}

This report gives a brief summary of the performance and output of the accelerator complex and associated accelerator-based experiments during FY 2016. It therefore summarizes the work of many people from Fermilab and from the collaborating institutions. The credit for the successful outcome of the FY 2016 running is shared amongst many. 